\title{
Análisis funcional de los restos líticos tallados de la cueva de Praileaitz I (Deba, Gipuzkoa)
}

\author{
Praileaitz I haitzuluko (Deba, Gipuzkoa) tailatutako harri-aztarnen analisi funtzionala \\ Use-wear analysis of knapped lithic tools from Praileaitz I (Deba, Gipuzkoa)
}

PALABRAS CLAVE: Instrumentos líticos, Traceología, Magdaleniense, País Vasco.

GAKO-HITZAK: Harri-tresnak, Trazeologia, Madeleine, Euskal Herria.

KEYWORDS: Lithic Tools, Use-wear Analysis, Magdalenian, Basque Country.

Ignacio CLEMENTE CONTE(1), Virginia GARCíA DÍAZ ${ }^{(2)}$, Assumpció VILA MITJÀ(1)

\section{RESUMEN}

En este trabajo presentamos los resultados del análisis funcional aplicado a los restos líticos del nivel "Magdaleniense Inferior" de Praileaitz I. Los rastros de uso nos han permitido discernir qué restos líticos fueron utilizados como instrumentos de trabajo y en qué actividades productivas pudieron intervenir. Es de destacar el uso de un alto porcentaje (60\%) de esos instrumentos para el aprovechamiento de recursos animales que abarcan desde la obtención de los mismos, con probables elementos de proyectiles, el aprovechamiento de la carne como recurso alimentario hasta la explotación de pieles y huesos como materia prima para la manufactura de otros bienes de consumo.

\section{LABURPENA}

Lan honetan Praileaitz I-eko Behe Madelein mailako harrizko aztarnen analisi funtzionalaren emaitzak aurkezten ditugu. Erabilera-arrastoek ahalbidetu digute bereizten zer aztarna litiko erabili zituzten lanabes moduan eta, gainera, zer jardueratarako baliatu zituzten. Tresna horietako ehuneko altu bat (\% 60) abere-baliabideen ustiapenean erabili zirela nabarmentzen da. Horien barnean sartzen dira: animaliak lortzeko erabiliak, eta horien artean ehiza ustezko jaurtigai-elementuak; elikadura-baliabide bezala haragia aprobetxatzeko erabiliak; eta baita beste kontsumo-ondasun batzuen manufakturarako larru eta hezur lehengaien ustiapena egiteko erabiliak ere.

\section{ABSTRACT}

In this work the results of the functional analysis applied to the lithic remains from the "Lower Magdalenian" level of Praileaitz I are presented. Use-wear traces allowed the identification of the lithic remains used as work tools as well as the production activities in which they took part. It is outstanding that a high percentage (60\%) of those instruments was used for working animal resources. This included hunting, made evident by the presence of probable projectiles elements, the use of meat as food resource, and the exploitation of hides and bones as raw material for the manufacture of other goods.

\section{1.- INTRODUCCIÓN}

Entre el material arqueológico recuperado en el suelo de ocupación del Magdaleniense Inferior de Praileaitz I destacan unos cantos de piedra perforados -colgantes- con una serie de incisiones o grabados que han sido considerados como evidencias de arte mobiliar (PEÑALVER, MUJIKA, 2003). La hipótesis más plausible era que los grabados y perforaciones en esas piezas fueran realizados con algún instrumento lítico, tal y como sugieren las investigaciones y experimentaciones de otros investigadores (p.e., D'ERRICO, 1988a y b, 1991). Se nos solicitó analizar microscópicamente los restos líticos recuperados en el mismo suelo de ocupación para comprobar si entre ellos podíamos identificar alguno que hubiera sido utilizado para realizar esas labores. A pesar de que las superficies de los restos líticos de ese nivel pre- sentan una fuerte alteración postdeposicional (cf. infra) que impide un exhaustivo análisis microscópico, hemos podido determinar que ninguno de esos restos líticos fue utilizado para el trabajo de rocas así como identificar otra serie de actividades productivas llevadas a cabo con los mismos.

Según X. Peñalver y J.A. Mujika los materiales recuperados en el nivel Magdaleniense Inferior se localizan "...en un medio sedimentológico arcilloso, que en ocasiones contiene cantos o concreciones estalagmíticas espacialmente localizadas, muy homogéneo en toda su potencia hasta el suelo de cantos calizos..." (PEÑALVER, MUJIKA, 2003: 106). Pensamos que este proceso sedimentario de acumulación de arcillas juntamente con la humedad y carbonatos procedentes del sistema cárstico de la propia cavidad, así como el suelo de cantos

\footnotetext{
(1) Departamento de Arqueología y Antropología. Institución Milá y Fontanals, CSIC. Egipcíaques 15. 08001 Barcelona. ignacio @imf.csic.es (2) Faculty of Archaeology, Leiden University, Leiden, the Netherlands.
} 
calizos, ha contribuido a que las superficies de los sílex tallados hayan sufrido determinadas alteraciones postdepositacionales que han supuesto una dificultad añadida al análisis microscópico ya que han podido eliminar, o por lo menos enmascarar, ciertos rasgos microscópicos de los rastros de uso. Estas alteraciones se presentan en forma de pátinas, a lo que hay que añadir pulidos debidos al movimiento y contacto con otros ítems líticos, como los pulidos tipo "G" (MOSS, 1983), y lustres de suelo tan desarrollados que en algunos casos podrían llegar a confundirse incluso con pulidos debidos al trabajo de materias duras ${ }^{1}$ (hueso, rocas, etc.). Sin embargo, es de destacar que el movimiento de los restos líticos en el sedimento no ha sido brusco ni muy acentuado, pues de haberlo sido las aristas y filos estarían más dañados; se habrían mellado más (especialmente las zonas finas -más débiles-), presentarían redondeamientos más acentuados, etc. Este hecho ha permitido diferenciar con mayor claridad los filos activos de los instrumentos y determinar específicamente aquellos restos que no fueron utilizados. Tan solo un par de piezas se han considerado como no analizables microscópicamente (NAM) debido a las alteraciones y, a pesar de ello, una (la laminilla P.A.12B.273.5) se considera como de uso posible (PO) por las características morfométricas de las melladuras presentes en su filo derecho.

\section{2.- DETERMINACIÓN DE LOS INSTRUMENTOS DE TRABAJO LÍTICOS Y LAS ACTIVIDADES LLEVADAS A CABO EN PRAILEAITZ I}

Entendemos como instrumento de trabajo lítico todo aquel resto que presenta en su superficie rastros de haberse utilizado como tal (CLEMENTE 1997, BRIZ et al. 2005). En el nivel Magdaleniense Inferior de Praileaitz I éstos suponen el 33\% (17 de los 52 analizados) del material lítico recuperado en el vestíbulo y en la primera sala interior de la cueva. La gran mayoría de los restos líticos son de sílex, pues tan solo tres lascas son de caliza. Aunque en este nivel que nos ocupa no se haya recuperado ningún núcleo, esto no quiere decir que no se haya llevado a cabo en el sitio ninguna actividad de producción lítica. La presencia de elementos corticales o semicorticales, especialmente de lascas y fragmentos, así lo podría estar indicando. Tal vez el área donde se pudo llevar

\footnotetext{
${ }^{1}$ El que estas alteraciones microscópicas no vayan acompañadas de otros estigmas de uso como el redondeamiento y orientación del filo, presencia de melladuras en los filos agudos, etc., ayuda a discernirlas como tales y no confundirlas con rastros de uso; aunque bien es cierto, tal y como hemos registrado en otros yacimientos (CLEMENTE, en prensa; CLEMENTE, PIJOAN, 2005), que estas alteraciones han podido enmascarar las huellas microscópicas de uso poco desarrolladas y especialmente las relacionadas con el trabajo de materias blandas animales. De ahí que en estos casos el estudio de los rastros de uso en su conjunto, y especialmente los macroscópicos, ayudan a determinar su uso y plantear hipótesis sobre los procesos productivos en los que intervinieron.
}

a cabo esta actividad ha quedado fuera de la superficie revisada en este estudio, o podría haberse ubicado en los alrededores de la entrada a la cueva. De los 52 restos analizados, los soportes básicos más representados son las lascas (23), seguido de laminillas (20) y láminas (8)². Además, hemos clasificado un pequeño resto lítico que correspondería con el positivo de un "golpe de buril", por lo que también queda patente el estadio de formación de los instrumentos a partir de los soportes explotados durante la producción lítica (PIÉ, VILA, 1991). Aunque el color gris y blanco-gris sea el predominante, es remarcable la explotación de un sílex marrón más translúcido para la manufactura específica de laminillas y particularmente laminillas de dorso. Probablemente los nódulos de este sílex destinado a la manufactura de estos soportes fueran también de un tamaño menor que los utilizados para las láminas. Las lascas y fragmentos de sílex pueden estar relacionadas con la preparación de los núcleos para láminas y laminillas, y, en algunos casos (5 de 23), los soportes más idóneos fueron aprovechados para utilizarlos como instrumentos de trabajo. En cuanto a los otros productos líticos hay que destacar que la mitad (4) de las láminas presentan rastros de uso y que los soportes que probablemente presenten más indicios de utilización son las laminillas y, más concretamente, las laminillas de dorso (Fig. 1).

En cuanto a los materiales trabajados con los instrumentos líticos con uso seguro y probable (17, con 20 filos utilizados) es remarcable el alto porcentaje (al menos el 60\%) utilizado para el aprovechamiento y explotación de recursos animales (Fig. 2). Entre estos instrumentos agrupamos tanto a aquellos que probablemente fueron destinados a la adquisición de masa animal (caza) como son las "laminillas de dorso", concretamente las de menor tamaño (cf. infra), que pudieron ser utilizadas como elementos de proyectil (20\%), como a los utilizados para el procesamiento de recurso animal blando (carne y/o piel - que suponen el 35\% de los instrumentos identificados) y los que han trabajado sobre materia dura animal, hueso y/o asta ( 5\%).

Como se puede observar en la primera figura, las laminillas, y específicamente las laminillas formadas por un borde abrupto o muy abrupto -"laminillas de dorso"-, son los restos que consideramos han sido utilizados en mayor porcentaje relativo.

Pero antes de continuar con estos instrumentos de Praileaitz I, y realizar una descripción de cómo se utilizaron los distintos instrumentos según los soportes básicos (láminas, laminillas y lasca) vamos exponer las distintas hipótesis y propuestas sobre el uso de los microlitos y

\footnotetext{
2 En estos grupos se incluyen también los fragmentos. Para delimitar métricamente entre láminas y laminillas hemos seguido la opción planteada por lbáñez y González al estudiar los materiales de Laminak II (1994, p. 87). Esta delimitación se basa especialmente en la anchura de estos productos considerando como laminillas -aquellas que tienen menos de $12 \mathrm{~mm}$ de ancho y que, generalmente no sobrepasan los $40 \mathrm{~mm}$ de largo.
} 


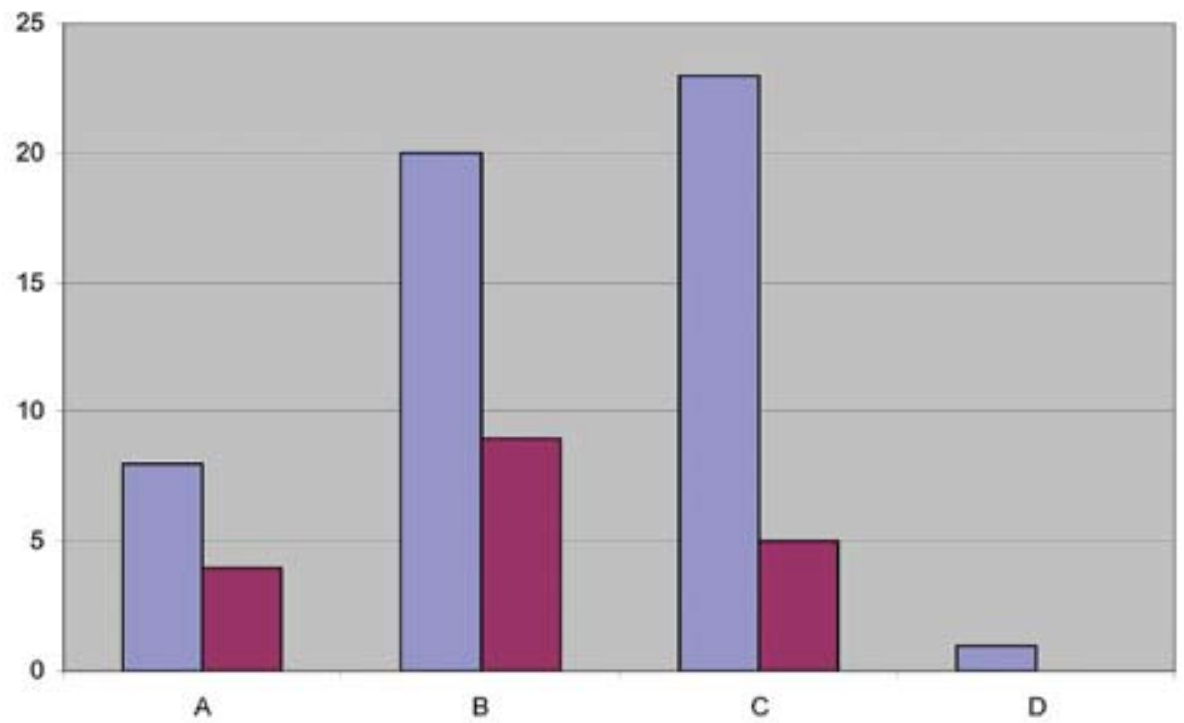

Fig. 1. Praileaitz I - A: láminas y fragmentos; B: laminillas y fragmentos.; C: lascas y fragmentos; D: Otros ("positivo de golpe de buril"). La columna de la izquierda marca el número de efectivos y la de la derecha representa los utilizados como instrumentos de trabajo. / Praileaitz I- A: Blades and blade fragments; B: bladelets and bladelet fragments; C: flakes and flake fragments; D: Others (iburin spalii). The left column shows the total amount of implements while the right column shows the amount of implements with traces of wear.

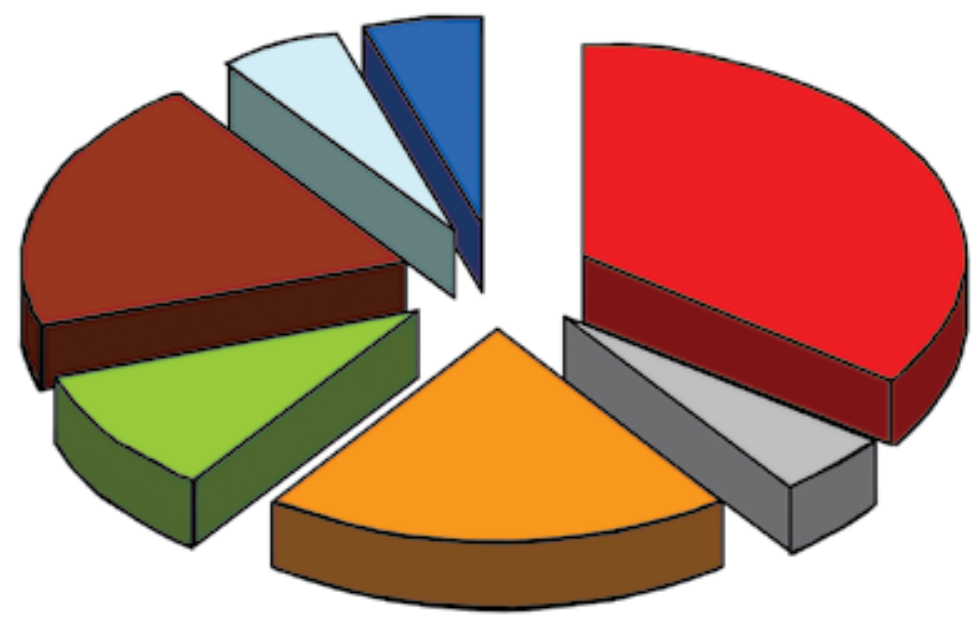

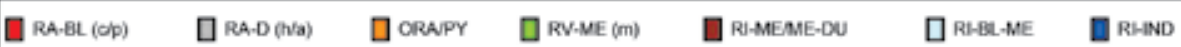

Fig. 2. Relación de materias trabajadas. RA-BL (c/p) = Recurso Animal Blando (carne/piel); RA-D (h/a) = Recurso Animal Duro (hueso/asta); ORA/PY = Otro Recurso Animal/Proyectil; RV-ME $(\mathrm{m})=$ Recurso Vegetal Medio (madera); RI-ME/ME-DU = Recurso Indeterminado Medio/Medio-Duro; RI-BL/ME = Recurso Indeterminado Blando/Medio; RI-IND = Recurso Indeterminado-Indeterminado. / Worked materials. RA/BL $(\mathrm{c} / \mathrm{p})=$ soft animal material (meat/hide); RA-D (h/a) = Hard Animal Material (bone/ antler); ORA/PY = Other animal materials/Projectile; RV-ME $(\mathrm{m})=$ Medium Vegetal Material (wood); RIME/ME-DU = Undetermined Medium Material/Medium-Hard; RI-BL/ME = Undetermined Soft/Medium Material; RI-IND = Undetermined Material-Undetermined. 
"laminillas de dorso" que hemos recopilado en bibliografía especializada. Como se podrá observar, muchas de estas hipótesis no tienen ningún fundamento científico; otras, como las que se basan en análisis de los rastros de uso, sí.

\section{3.- SÍNTESIS HISTORIOGRÁFICA SOBRE EL USO DE INSTRUMENTOS MICROLÍTICOS EN GENERAL $Y$ “LAMINILLAS DE DORSO" EN PARTICULAR}

Los instrumentos microlíticos, al igual que el resto del instrumental lítico, han recibido un sinfín de atribuciones funcionales que por lo general, hasta la aplicación del método propuesto por S.A. Semenov, no tuvieron ningún fundamento científico. Es tan solo a partir del análisis de los rastros o huellas de uso, denominado "traceología" por su creador a mediados del siglo pasado, cuando se ha podido determinar científicamente el uso de los instrumentos de trabajo usados por las diferentes sociedades prehistóricas (SEMENOV 1957/64/81). Así pues, podemos encontrarnos con trabajos como el del soviético V.A. Gorodtsov (1923) en el que proponía que los instrumentos microlíticos sirvieron para tatuarse el cuerpo y para la realización de tareas de peluquería como el afeitado, así como para perforar pieles delgadas y coserlas. Más tarde, J. Verheyleweghen pretendió reafirmar la hipótesis planteada anteriormente por el investigador belga Cristian Ofoven, según la cuál las laminillas de dorso deberían haber servido como limas o raspadores. Para demostrarlo realizó una serie de experimentos (sin ningún tipo de control ni observación microscópica) para manufacturar con esos instrumentos unos arpones de hueso y compararlos luego con materiales arqueológicos: "un estudio minucioso de todo el instrumental lítico nos lleva a la conclusión que sólo las laminillas de dorso podrían realizar esta función" (VERHEYLEWEGHEN, 1951: 358). Él las denominó "limas magdalenienses" y afirmó que estaban predestinadas para trabajos sobre hueso, marfil o cornamentas de animales. Otros investigadores las denominan "navajas" ya que consideran que generalmente sirvieron para cortar aunque también pudieron utilizarse para realizar tatuajes o para cortar el pelo (CHEYNIER 1953). En este último trabajo A. Cheynier cita a otros prehistoriadores como D. Peyrony y A. Breuil, los cuales sostenían que las laminillas de dorso eran utilizadas como elementos de armas arrojadizas, sujetándolas con ayuda de resinas y encajadas en soportes de madera u óseos, aunque también pudieron servir como elementos de hoces y arpones (CHEYNIER, op. cit.). P.I. Boriskovski señala la abundante presencia de puntas realizadas en finas y estrechas laminillas en el yacimiento de Ambrosiev. Él considera que sirvieron para la fabricación (bien en hueso o madera) de los extremos de armas de caza arrojadizas o arpones (cit. en ROGACHEV, 1955: 43). A.N. Rogachev las relaciona con la pesca, pero también admite otras posibilidades: "como mostraron nuestras observaciones, parte de ellas (sobre todo las puntas), puntas de tipo "gravette" y algunas otras, podrían ser utilizadas como instrumentos punzantes o cortantes; "usándolas, incluso, sin enmangue para coser los cueros y pieles de animales" (ROGACHEV 1955 p. 132). Sin embargo, el tamaño y fragilidad de estos instrumentos dan pie a considerar la necesidad de algún tipo de enmangue para su utilización. Así por ejemplo N.D. Praslov (1972: 72) plantea que: "por sí solos, sin estar sujetos a un mango, no podrían utilizarse por sus medidas. Un pequeño retoque de los filos, dándoles una forma recta y reforzando los bordes, y también el disminuir la anchura al retocar ambos filos nos muestra que estos útiles los colocaban en ranuras laterales de tales instrumentos como puntas de lanza o cuchillos". Este mismo autor considera que las puntas pequeñas las usaban para grabar (o ranurar) hueso y posiblemente madera. "El estudio de los rastros de grabado en huesos, recogidos en Muralova, muestra que gran parte de las líneas, que forman la composición, se han hecho con instrumentos muy finos. Con buriles estas líneas no se pueden realizar y con puntas microliticas si... Los rastros de uso lineales observados por V.E. Shchelinsky en el vértice de una punta se formaron, seguramente, por este tipo de trabajo" (PRASLOV; 1972: 72).

Según A.K. Kozlowski y B. Ginter (1975), basándose en el hallazgo de una mandíbula de ciervo con una punta incrustada en un yacimiento magdaleniense de Bélgica $^{3}$, plantea que las piezas de dorso se pudieron utilizar de diversas formas, bien como cuchillos o como puntas de armas arrojadizas (lanzas/flechas). G. Cauvin (1982) con relación a los microlitos escribió que, según los análisis de G. Odell en el yacimiento de Bergumermeer (Holanda), no sólo las puntas microlíticas sino todos los geométricos de distintos tipos, al igual que las laminillas de dorso y numerosos microlitos de distintas morfologías fueron utilizados como elementos de armas arrojadizas. G. Odell (1978) ya con análisis funcionales señala que la mayoría de las piezas tipo "laminillas de dorso" de Bergumermeer muestran los mismos caracteres funcionales que los considerados morfológicamente como "puntas". De todas formas entre las puntas de este yacimiento pudo determinar que se utilizaron como cuchillos, agujas o buriles. Aplicando también el análisis funcional, H. Plisson (1985) estudió las laminillas de dorso de Pincevent (Francia). De las 91 laminillas, 21 (23\%) presentaban rastros de haberse utilizado como elementos de armas arrojadizas y 2, posiblemente habían sido utilizadas como cuchillos.

En este mismo trabajo analiza las laminillas, laminillas de dorso y puntas de dorso de otro yacimiento del $\mathrm{Pa}$ leolítico Superior, Andernaj. De las 52 laminillas solo 4 tienen rastros de uso (3 para cortar piel y 1 como elemento de un arma arrojadiza). De las 9 laminillas de dorso 5

\footnotetext{
${ }^{3}$ Tal y como queda reflejado en los trabajos de D. Nuzhnyj (1989, 1990, 2000), el hallazgo de microlitos y laminillas de dorso, tanto enmangados en sus astiles como incrustados en restos faunísticos y humanos, es bastante común a lo largo de toda la prehistoria.
} 
presentan rastros de uso: 3 son elementos de un arma arrojadiza, y en 1 se observan estrías indiferenciadas en el filo y "rastros longitudinales de choque", característicos de las armas arrojadizas. La quinta laminilla se utilizó para cortar piel. De este mismo yacimiento se analizaron 25 puntas de dorso. Once de ellas presentan rastros de uso: 5 ó 6 como componentes de armas arrojadizas; 5 ó 6 como cuchillos para carne-piel.

Para E. Moss y M. Newcomer (1982) las laminillas de dorso funcionaban como cuchillos, perforadores o puntas de lanzas. Elaboraron 16 lanzas experimentales que clavaron sobre cuerpos de animales y no se formaron rastros de uso. También realizaron 13 experiencias de perforación y sólo en la mitad observaron rastros de uso. Estos autores consideran que las laminillas de dorso eran elementos de útiles compuestos, y se pueden considerar como cuchillos si presentan micropulido de carne. En las armas arrojadizas no se suelen formar rastros de uso, aunque estén utilizadas y si presentan algún tipo de rastro se trataría de una reutilización.

También a través del análisis funcional de materiales del sitio Shirokii Mis, yacimiento del Paleolítico Superior al NO del Cáucaso (CLEMENTE, 1989), observamos que entre las laminillas de dorso las habían sin ningún rastro de uso, y en otros casos se determinó su uso como probables componentes de un filo usado como cuchillo para el corte de materia blanda animal (carne).

El análisis funcional para el estudio del uso en estos tipos de instrumentos también se ha aplicado a varios yacimientos del norte de la Península lbérica: Rascaño y el Juyo en Cantabria (KEELEY, 1988); Laminak II y Santa Catalina en Bizkaia y Berniollo en Araba (GONZÁLEZ, IBÁÑEZ, 1994b, IBÁÑEZ, GONZÁLEZ, 1997 y 1998). Los resultados comparativos entre varios yacimientos han servido para determinar las actividades económicas llevadas a cabo en ellos con los diversos medios de producción líticos estudiados, e inferir a partir de esos resultados la funcionalidad y el carácter del sitio. En el trabajo de L. Keeley (1988) se comparan los resultados de estos dos yacimientos de Cantabria con los de Verberie, otro yacimiento de la misma "cronología magdaleniense" en la cuenca parisina (Francia). Dejando de lado las diferencias tecnológicas entre el yacimiento francés y los del cantábrico, en parte debidas también a las características de las materias primas utilizadas, en cuanto a la funcionalidad, la diferencia de porcentaje entre piezas utilizadas para el trabajo de piel seca y fresca así como entre los instrumentos usados para hueso/asta y los elementos de proyectil, le permite distinguir entre "campamento base" (caso del Juyo) y "estaciones de caza" (Verberie y Rascano) (KEELEY, 1988, p. 24). Para L. Keeley según los resultados de la correlación entre tipología y uso, la gran mayoría (82-90\%) de las laminillas de dorso fueron utilizadas como elementos de proyectiles (KEELEY, 1988: 22).

En cuanto a los yacimientos analizados en el País Vasco por J.J. Ibáñez y J.E. González, los conjuntos más antiguos- el nivel magdaleniense de Santa Catalina y Laminak II- son los que proporcionan indicios de estrategias menos planificadas y los más modernos- el nivel aziliense de Santa Catalina y Berniollo- reflejan una planificación de actividades destinadas por una parte a la captura de animales y, por otra, al procesado de esos animales con las actividades específicas que ello implica (IBÁÑEZ, GONZÁLEZ, 1997: 294 y 1998). Por otra parte, estos mismos autores, al analizar las laminillas de dorso observan que se pueden dar diversos casos y diferencian tres grupos. Primeramente las laminillas que no presentan ningún tipo de huella de uso; en segundo lugar aquellas en las que se observan rastros de impacto, ya sea en forma de estrías o fracturas y, por último, aquellas que presentan rastros de haber trabajado materias blandas animales (piel o carne) (GONZÁLEZ, IBÁÑEZ. 1994b: 118). Éstas últimas suelen ser de mayor tamaño que las utilizadas como proyectiles y no en toda pieza lítica utilizada como elemento de proyectil o como punta de flecha se forma rastros debidos a su uso como tales (MOSS, NEWCOMER, 1982; MOSS, 1983; FISCHER et al., 1984; GONZÁLEZ, IBÁÑEZ, 1994a).

\section{4.- USO DE LAMINILLAS Y "LAMINILLAS DE DORSO" DE PRAILEAITZ I}

El 38\% del total de los restos recuperados en este nivel estudiado son laminillas. La mayoría (13) no están formadas con ningún tipo de retoque y casi todas están completas, tan solo hemos identificado un ejemplar como fragmento de laminilla. Las siete restantes presentan uno de los laterales formado por un retoque abrupto con tendencia a muy abrupto configurando lo que comúnmente se viene denominando "dorso". Es destacable que entre las trece laminillas sin retoque tan solo hemos podido identificar una como un instrumento "seguro" y otra como "uso posible". Esta última presenta un mellado irregular en su filo derecho, sin embargo las alteraciones postdepositacionales impiden la observación microscópica y no se puede identificar ni el movimiento ni la materia trabajada. La laminilla que presenta rastros de uso (P.A.8E.269.24 /113/), resultó utilizarse como perforador sobre un material indeterminado de dureza media/dura (¿madera, hueso?). Se utilizó la parte proximal de la laminilla como zona activa del instrumento; allí es donde tiene las melladuras en ambos laterales, reflejando un movimiento rotatorio. Pensamos que fue utilizada muy poco tiempo, tal vez para un trabajo puntual, ya que las aristas de las melladuras no llegan a embotarse y por lo tanto no se forma un micropulido diagnóstico de la materia trabajada.

Al analizar las laminillas de dorso (Fig. 3) llama la atención la diferencia de tamaño entre ellas. Igual que ocurre en otros yacimientos (p.e Laminak II, GONZÁLEZ, IBÁÑEZ, 1994b) se podría considerar la división en dos grupos tanto por su longitud como por las características microscópicas de sus filos. Las laminillas más largas (3) presentan en los filos no retocados una serie de pequeñas melladuras bifaciales y un ligero redondeamiento; atributos que se podrían relacionar con el corte de una 


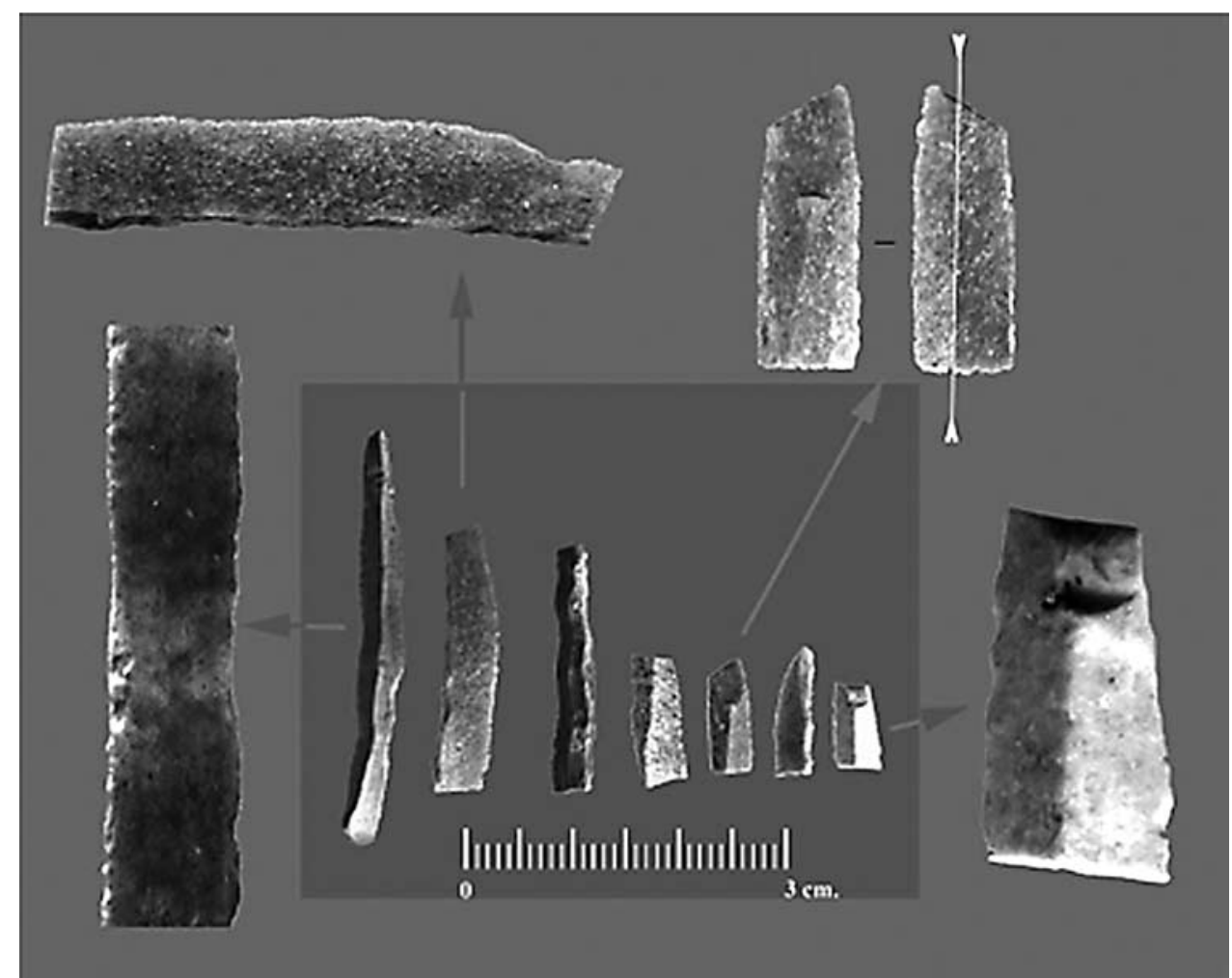

Fig. 3. Laminillas de dorso. Diferenciación en grupos por tamaños: las tres de la izquierda, de mayor tamaño-relacionadas con el corte de materia blanda animal (carne) y las cuatro más pequeñas -probables elementos de proyectil, dos de ellas con posibles fracturas de impacto. / Backed bladelets grouped by size: the three bladelets on the left, the bigger ones, were used to cut a soft animal material (meat); the other four bladelets, smaller, were possibly used as projectiles, two of them showing possible impact fractures.

materia blanda de origen animal, por lo que planteamos la probabilidad de una utilización para labores de descarnado o carnicería. La distribución de estos rastros de uso en el filo contrario al retocado nos estaría indicando que al filo retocado se le da forma de esa manera para lograr una mayor efectividad en su sujeción al mango, pues se logra una mayor superficie de contacto con la almáciga que va a servir de elemento de unión entre el soporte lítico y el mango.

Las otras cuatro laminillas, de menor tamaño, carecen de melladuras en sus filos. Dos de ellas presentan fracturas reflejadas que podían ser producto de un impacto (FISCHER et al., 1984; NUZHNYJ, 1990; GONZÁLEZ, IBÁÑEZ, 1994a, etc.). Otra presenta, junto al borde no retocado, una "playa de abrasión" con un pulido compacto, brillante y rugoso, y estrías orientadas paralelas al filo, que también podría ser debido al uso como proyectil. La última no presenta ningún signo claro de uso como tal, aunque como ya hemos visto más arriba, esto no significa que no pudiera haber sido utilizada como elemento de proyectil. Como ocurre con las otras laminillas de dorso, en este caso el borde retocado también habría sido la parte enmangada. Además, una de las laminillas con la probable "fractura de impacto" presenta en su superficie una diferencia clara de coloración entre un lado y otro.
Tal vez esta alteración diferencial podría estar indicándonos hasta donde llegaba el contacto con la almáciga y/o el enmangue (Fig. 3 derecha superior).

Así pues, las laminillas de dorso podrían haber servido para componer dos clases de instrumentos distintos. Por una parte, las más grandes podrían haber sido elementos de cuchillo (de filo compuesto por varias piezas) utilizados para actividades de carnicería y, por otra, los elementos más pequeños podrían haber sido usados como elementos de armas arrojadizas, para actividades cinegéticas.

Esto mismo ha sido observado también en otros yacimientos del Paleolítico Superior del País Vasco (GONZÁLEZ, IBÁÑEZ, 1994b).

\section{5.- USO DE LAS LÁMINAS}

El 50\% de los productos laminares (láminas y fragmentos) presentan rastros de haberse utilizado como instrumentos de trabajo (Fig. 1). Dos de estas láminas fueron utilizadas para procesar algún recurso animal blando o blando/medio, en un caso P.A.10F'.329.17 (13731) la materia cortada con ese fragmento medial de lámina no ha sido determinada y en el otro, P.A.16G'.346.2 (12967), 


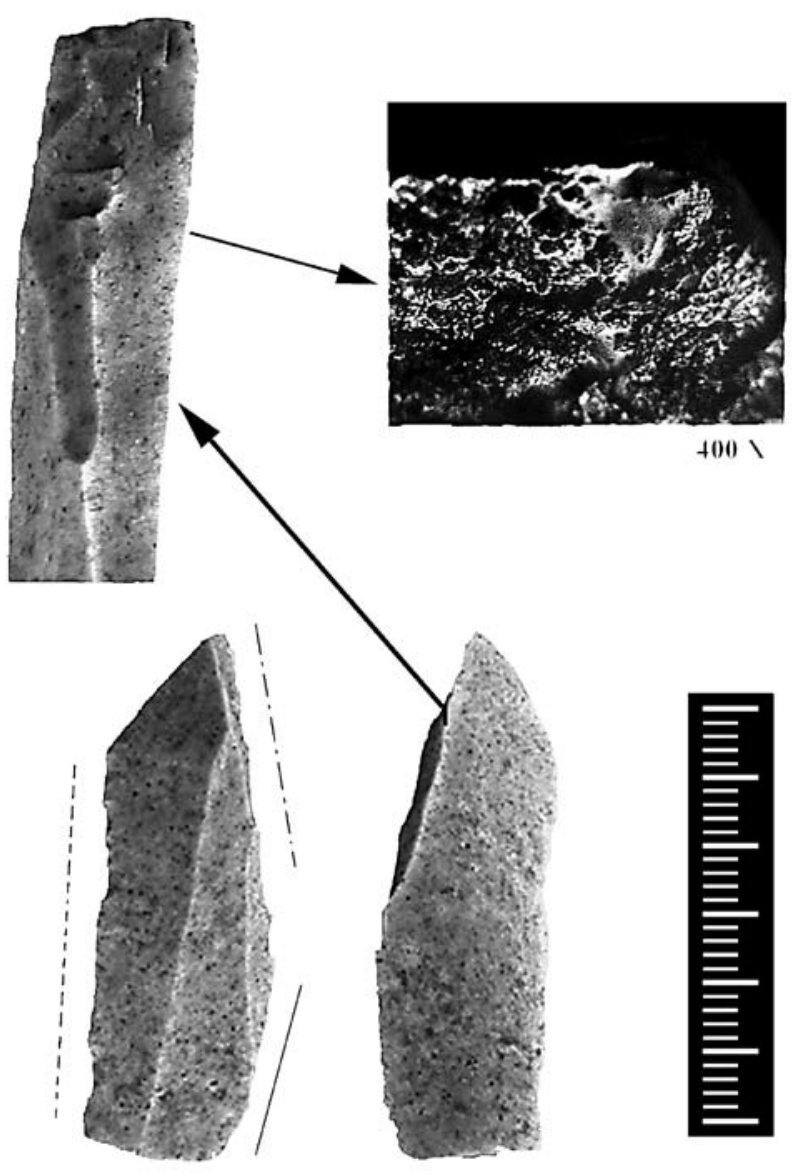

Fig. 4. Lámina-buril con rastros de uso en ambos filos. La foto superior muestra el micropulido observado en la cara ventral del filo derecho o faceta del buril y atribuido al trabajo de una materia dura animal (¿hueso, asta?). / Blade/Burin displaying use-wear traces on both edges. The upper picture shows the micro-polish displayed on the ventral surface of the right edge, or burin facet, related to processing a hard animal material (bone?antler?).

también han sido utilizados ambos filos en una acción longitudinal de corte, y la materia trabajada ha sido la piel. Llama la atención el acentuado redondeamiento que presenta una parte de las aristas dorsales, que hemos atribuido a una presión ejercida, probablemente con la propia mano, al sostener el instrumento durante su uso.

La lámina P.A.10G.256.10 (193), presenta rastros de haber estado en contacto con un material de dureza media/dura en la parte proximal del filo derecho. Por la distribución del pulido y las estrías el movimiento fue tanto longitudinal como transversal, en una acción relacionada con el adelgazamiento o desbastado de una materia tipo madera. Las alteraciones postdepositacionales han afectado también a la superficie con micropulido de uso y hace que determinadas características de éste no puedan ser totalmente reconocibles.

Por último, la lámina P.A.10F.271.3 (164), formada por un "retoque de buril", ha resultado ser el instrumento de trabajo con mayor amortización, pues ambos filos presentan rastros de uso, y en el caso del filo derecho utilizado para actividades diferentes. Así pues, el filo izquierdo fue utilizado en una acción transversal (raerraspar) sobre una materia indeterminada de dureza media/dura; mientras que el filo derecho fue utilizado en su parte proximal para cortar una materia indeterminada de dureza blanda/media y en su parte distal, lo que correspondería con el lateral del buril, en una acción transversal sobre una materia animal dura -hueso/asta- (Fig. 4). El ápice del buril presenta unos negativos de pequeñas extracciones que parecen más relacionados con la manufactura del "golpe de buril" que con un uso, pues no se acompañan de ningún otro rastro (embotamiento-redondeamiento del filo, estrías, micropulido...). Es el lateral derecho del buril el que presenta rastros de uso como instrumento, en una acción transversal apoyando la cara ventral en el material trabajado. En este caso, aparte del micropulido marginal, de trama compacta, liso, brillante y con "resquebrajaduras", también se forman una serie de melladuras escalonadas relacionadas con el contacto de una materia dura.

\section{6.- USO DE LAS LASCAS}

Tan sólo hemos observado rastros de uso en 5 de las 23 lascas del nivel estudiado. Las lascas elegidas para utilizarlas como instrumentos de trabajo presentan cualidades morfométricas -tamaño, ángulo del filo, forma, etc.-, que las hacen idóneas para los procesos de producción en los que intervinieron. Así pues, utilizaron dos lascas alargadas con ángulos de filo entre 20 y 30 grados para cortar dos materias distintas. El filo izquierdo de la lasca P.A.20G'.348.1 (12922), sirvió para cortar materia animal blanda indeterminada (carne/piel fresca...) y la lasca P.A.6C.276.2 (34) se utilizó para cortar un recurso vegetal de dureza media (probablemente madera). Ésta última es, curiosamente, uno de los soportes más

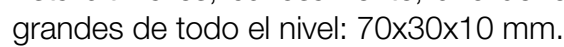

Las alteraciones postdepositacionales, al igual que ha ocurrido con el resto de los soportes, han impedido realizar un análisis óptimo de las superficies de los sílex, no siendo posible reconocer en muchos casos la materia concreta trabajada; sin embargo, hemos podido hacer una aproximación a la dureza del material trabajado en la mayoría de los casos, a excepción de la lasca P.A.20G'.344.2 (12972) con su filo izquierdo utilizado probablemente en una acción transversal. En otra lasca P.A.10G.260.13 (190), una "muesca" natural con un ángulo entre 50 y $60^{\circ}$, fue utilizada para trabajar, en una acción transversal (para rebajar o alisar) una materia indeterminada de dureza media a media/dura (¿tal vez madera?).

Finalmente, la lasca P.A.6A.289.96 (12005), presenta en su filo derecho rastros claramente atribuibles al contacto con un material abrasivo (Fig. 5). Por las características de los rastros la materia trabajada en este caso fue la piel/cuero, sin embargo el micropulido presenta carac- 

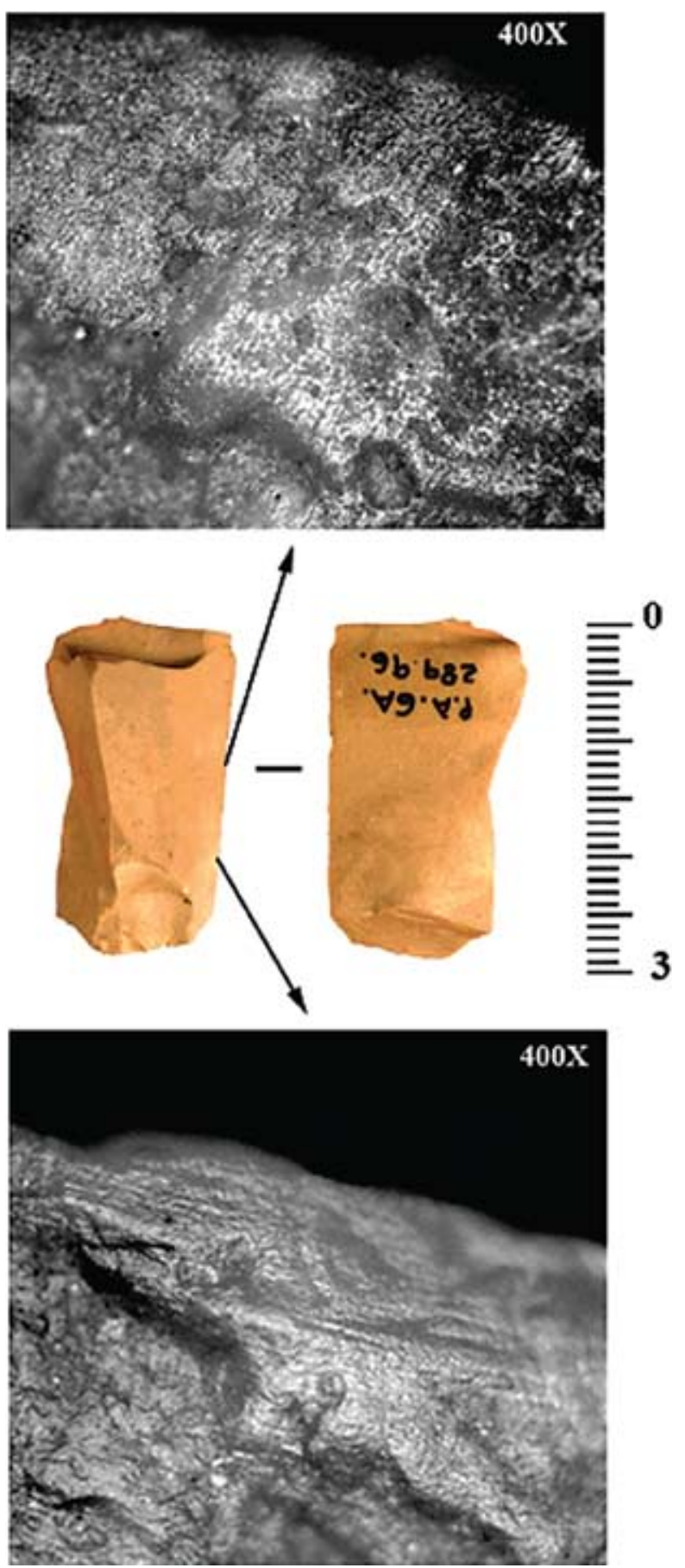

Fig. 5. Lasca utilizada para el corte de piel/cuero. En foto superior, las estrías y orientación del pulido muestran una cinemática transversal -presión inicial del corte y el resto del filo muestra una clara orientación longitudinal de corte (foto inferior). / Flake used for cutting hide/leather. The upper picture shows striations and a polish orientation related to a clear transversal action while the other part of the edge shows a clear longitudinal cutting action (lower picture). terísticas que podrían recordar al producido al trabajar piel con abrasivos (o pieles/cueros sucios, con partículas abrasivas). La actividad realizada por este instrumento muestra cierta complejidad. Por una parte, en la zona central del filo derecho observamos una estrecha área con micropulido que presenta una orientación claramente perpendicular al filo (foto superior Fig. 5), mientras que en el resto del filo las estrías y orientación del micropulido nos indican un movimiento claramente de corte, es decir paralelo al filo. Pensamos que ambas cinemáticas se podrían dar en una actividad destinada a la obtención de tiras finas de cuero, ya que la presión inicial con la parte central del filo, a la vez que se estira de la piel hacia los lados, permite iniciar un corte que se va dirigiendo y/o rectificando cuando aparece algún impedimento, al igual que se termina el corte con movimientos longitudinales.

\section{7.- DISCUSIÓN}

A partir del análisis funcional del material lítico tallado se ha podido determinar que en el nivel "Magdaleniense Inferior" de Praileaitz I se llevaron a cabo una serie de actividades relacionadas tanto con la subsistencia como con la producción y/o mantenimiento de bienes. Vemos pues que las actividades más representadas están relacionadas con el aprovechamiento de recursos animales (Fig. 2), desde el aprovisionamiento de este recurso a través de la caza -reflejada en las "laminillas de dorso" de pequeño tamaño utilizadas probablemente como elementos de proyectil; actividades relacionadas con procesos de carnicería o desmembramiento de animales -reflejadas en todos los tipos de soporte utilizados: laminillas de dorso de mayor tamaño, dos láminas y dos lascas. También ha quedado patente el aprovechamiento de determinadas partes de los animales (piel, hueso y/o asta) como materia prima para la confección de bienes de consumo: caso de la arista larga del buril usado para raspar una materia ósea y la lasca utilizada probablemente para la manufactura de tiras de cuero. Por las características específicas de los micro-rastros observados en este último instrumento (Fig. 5), la piel trabajada con él podría haber estado impregnada con algún tipo de abrasivo.

La localización de un alto porcentaje de las laminillas de dorso en la zona cercana al hogar y a la roca considerada como un "asiento" (cuadrículas 10F y 12D) podría estar relacionada con el acondicionamiento y/o reparación de los cuchillos compuestos y puntas de proyectil. La utilización del calor del fuego para conseguir un estado idóneo de la almáciga para el enmangue y desenmangue de las piezas ya ha sido propuesto por otros investigadores e investigadoras, ya que es común encontrarse estos tipos de componentes de instrumentos en áreas de hogares o cercanos a ellos (p.e. Plisson 1985, Moss 1983, entre otros). Este hecho explicaría la localización de las laminillas de dorso fracturadas en áreas cercanas a hogares, en aquellos casos que esos elementos lleguen aún enganchados al astil y éste sea aún aprovechable 
para la misma función. De todas formas, las laminillas de dorso fracturadas también podrían haber llegado en el interior del animal abatido y entonces se localizarían con mayor probabilidad en el lugar donde el animal fuera descuartizado (cf. Plisson y Geneste, 1989).

Son muy pocos los instrumentos identificados para trabajar otros recursos, como sería el caso de la madera, que en este caso suponen el $10 \%$ de los instrumentos, aunque existe otro $20 \%$ que podría haber trabajado tanto madera dura como materia ósea o asta, pero las alteraciones y/o el poco desarrollo de los micro-rastros no nos ha permitido determinar con exactitud la materia trabajada (Fig. 2).

\section{8.- CONCLUSIONES}

A pesar de las alteraciones microscópicas detectadas en las superficies de los restos líticos tallados de Praileaitz I, hemos podido identificar determinadas huellas de uso que nos han permitido entrever ciertas actividades productivas llevadas a cabo con los mismos. Tras el análisis funcional podemos afirmar que ninguno de los instrumentos de trabajo identificados presenta huellas de haber trabajado algún mineral, ni para grabar ni para perforar. Por lo tanto no hemos podido identificar los instrumentos que perforaron o realizaron incisiones en los "colgantes" de piedra recuperados en el mismo nivel arqueológico (PEÑALVER, MUJIKA, 2003). Ante esto nos podemos plantear nuevas cuestiones: ¿fueron manufacturados en el sitio?, si es así debió hacerse en otro lugar aún no excavado donde quedaron sepultados los instrumentos utilizados para ello. Y si no ¿se trata entonces de objetos aportados al sitio ya elaborados?

En cuanto a la producción lítica en Praileaitz I, se puede observar una clara preferencia por la búsqueda de filos largos y agudos a través de una talla laminar y preferentemente micro-laminar, aunque no se pueda determinar dónde se realizó esa actividad. La ausencia de núcleos y de otros residuos con características específicas de la configuración de los mismos entre los restos recuperados, no nos permite atestiguar una producción lítica en el sitio aunque algunos productos laminares y lascas muestren presencia de cortes.

Los instrumentos de trabajo líticos del nivel "Magdaleniense Inferior" de Praileaitz I, fueron utilizados básicamente para el trabajo o aprovechamiento de recursos animales; para la adquisición de masa animal (caza) y para los procesos de carnicería o desmembrado de los animales. Por las actividades productivas llevadas a cabo en el sitio, si siguiéramos las indicaciones planteadas por Ibáñez y González (1997: 293) se podría decir que el grupo que ocupó la cueva en ese momento planificaba sus actividades ya que existe un desequilibrio entre la proporción de las actividades de adquisición frente a las de transformación, no existe una continuidad en los procesos técnicos ya que no se documentan todas las fases de la producción y, además, podría darse el caso de que existiera una reserva de productos líticos brutos (p.e. las laminillas de dorso sin uso). Si siguiéramos el criterio de L.H. Keeley (1988), por la diferencia de actividades documentadas -mayor presencia de proyectiles, actividades relacionadas con carnicería y trabajos en piel fresca y poco trabajo sobre hueso/asta y piel seca- deberíamos caracterizar el sitio más como un lugar de caza que como un campamento base. Si esto fuera así, habría determinadas cuestiones que siguen sin resolverse: ¿qué papel juegan los "colgantes" en un sitio especializado en la caza y obtención de recursos?; si se tratara de un lugar de los denominados "de culto" ¿qué significan las actividades productivas evidenciadas en un sitio de esa índole? Lo que si queda patente es la existencia de una inversión de trabajo en la producción de instrumentos líticos, que han sido amortizados a través de su uso para la obtención de recursos y bienes subsistenciales, por una parte; y por otra, en la producción (o en el transporte) y quizás también en la producción de los elementos de donde pendían esos "colgantes", también amortizados y abandonados, pero en este caso no destinados a la subsistencia sino a la reproducción social. Ante esto surge la cuestión ¿en cuál de las dos fue "más importante" o "más rentable" o de "mayor valor" esa inversión de trabajo?

De todas maneras, no podemos o no debemos llegar a conclusiones con sólo el análisis de los restos líticos. Esperamos que con los resultados de otras analíticas y estudios de otros materiales y su interrelación se puedan proporcionar respuestas a estas cuestiones planteadas, y de no ser así, tal vez se generen nuevas cuestiones cuyas respuestas se puedan obtener no necesariamente en el estudio únicamente de Praileaitz I.

\section{9.- AGRADECIMIENTOS}

Queremos agradecer a dos revisores anónimos por las correcciones y sugerencias hechas al texto. Sin duda la versión final resulta más comprensible tras realizar dichas correcciones. Aunque el texto de este trabajo se escribió hace más de siete años pensamos que aún sigue siendo de actualidad y esperamos que su lectura despierte aún interés. Agradecemos a los editores de este volumen el haber mantenido este trabajo para su publicación.

\section{0.- BIBLIOGRAFÍA}

BRIZ, I., CLEMENTE, I., PIJOAN, J., TERRADAS, X., VILA, A

2005 Stone tools in ethnoarachaeological contexts: theoricalmethodologicas inferences, en TERRADAS, X. (ed.). Lithic Toolkits in Ethnoarchaelogical Contexts. 1-7. (BAR International Series; 1370).

CAUVIN, J.

1982 Typologie et fonctions des outils préhistoriques: apports de la tracéologie a un vieux débat. Travaux de la Maison de l'Orient 5, 259-274. 
CHEYNIER, A.

1953. Les lamelles à bord abattu. Autonomie, origine évolution et usages posibles. Bulletin de la Société Préhistorique Française 50(1), 81-85.

\section{CLEMENTE CONTE, I.}

1989 Análisis morfológico y funcional de la industria microlítica de Shirokii Mis (yacimiento del paleolitico superior a orillas del Mar Negro, al NW del Caucaso). Tesis de licenciatura, Universidad Estatal de Leningrado. Trabajo inédito, original en ruso.

1997 Los instrumentos líticos del Túnel VII: una aproximación etnoarqueológica. Treballs d'Etnoarqueologia II, CSICU.A.B. Madrid.

En prensa El "lustre de suelo" y la conservación de los micropulidos de uso. Resultados experimentales para una aplicación arqueológica: La Rogère 1, locus IV, en BRUNET, M. \& FOLGADO, M. (eds.). Le site moustérien de la Rogère 1 (A89-96/104). Document Final de Synthèse, AFAN.

CLEMENTE, I., PIJOAN, J.

2005 Estudio funcional de los instrumentos de trabajo lítico en el "Embarcadero del río Palmones", en RAMOS, J. \& CASTAÑEDA, V. (eds.). Excavación en el asentamiento prehistórico del Embarcadero del río Palmones (Algeciras, Cádiz). Una nueva contribución al estudio de las últimas sociedades cazadoras y recolectoras. 252-282. Fundación Municipal de Cultura de Algeciras y Universidad de Cádiz.

D'ERRICO, F.

1988a Study of upper paleolithic and epipaleolithic engraved pebbles, en SANDRA L. Olsen (ed.). Reprinted from Scanning Electron Microscopy in Archaeology. 169-184. (BAR International Series; 452).

1988b Lecture technologique de l'art mobilier grave. Nouvelles méthodes et premiers résultats sur les galets graves de Rochedane. L'Anthropologie 92 1, 101-122.

1991 La signification des galets gravés aziliens et le mythe des calendriers lunaires préhistoriques. Annales de la fondation Fyssen 5/6, 63-73.

FISCHER, A., HANSEN, P.V., RAMUSSEN, P.

1984 Macro and Micro Wear Traces on Lithic Projectile Points. Experimental Results and Prehistoric Examples. Journal of Danish Archaeology 3, 19-46.

GONZÁLEZ URQUIJO, J.E., IBÁÑEZ ESTÉVEZ, J.J.

1994a Metodología de análisis funcional de instrumentos tallados en sílex. Cuadernos de Arqueología 14

1994b Análisis funcional del utillaje en sílex en el yacimiento de Laminak II. Kobie (Serie Paleoantropología) XXI, 111-129.

GORODTSOV, V. A.

1923 Arjeologia. Kamenni period. Moskva.

IBÁÑEZ ESTÉVEZ, J.J., GONZÁLEZ URQUIJO, J.E.

1994 La fabricación del utillaje lítico tallado en Laminak II. Kobie Paleoantropología XXI, 85-110.
1997 Diversidad funcional de los asentamientos del Paleolítico Superior. Una perspectiva desde el utillaje lítico, en DE BALBÍN, R. y BUENO, P. (eds.). // Congreso de Arqueología Peninsular, Tomo I- Paleolítico y Epipaleolítico. 287 296. Fundación Rei Afonso Henriques. Zamora.

1998 The production and use of lithic tools at the end of the upper palaeolithic in the Basque country, in MILLIKEN, S. (ed.). The Organization of Lithic Technology in Late Glacial and Early Postglacial Europe. 17-37. (BAR International Series; 700)

KEELEY, L.H.

1988 Lithic Economy, Style and Use: A Comparison of Three Late Magdalenian Sites. Lithic Technology 17(1), 19-25.

KOZLOWSKI, J.K., GINTER, B.

1975 Técnica de la talla y tipología de los instrumentos líticos. Universidad de la Habana, Facultad de Ciencias, Escuela de Ciencias Biológicas y Museo Antropológico «Montané ». La Habana.

MOSS, E.H.

1983 The functional analysis of flint implements. Pincevent and Pont d'Ambon: two cases studies from the french final Palaeolithic. (BAR International Series; 177).

MOSS, E.H., NEWCOMER, M.H.

1982 "Reconstruction of Tools Use at Pincevent: Microwear and Experiments". Studia Praehistorica Belgica 2, 289-312.

NUZHNYJ, D.

1989 L'utilisation des microlithes géométriques et non géométriques comme armatures de projectiles. Bulletin de la Société Préhistorique Française 86/3, 88-96.

1990 Projectile Damage on Upper Paleolithic Microliths and the Use of Bow and Arrow among Pleistocene Hunters in the Ukraine, en The interpretative possibilities of Microwear Studies, AUN 14, 113-124, Societas Archaeologica Upsaliensis. Uppsala.

2000 Development of Microlithic Weapons in the Stone Age, en BELLIER, C.; CATTELAIN, P. \& OTTE, M. (dirs.). La chasse dans la Préhistoire. 95-101. (ERAUL; 51).

ODELL, G.H.

1978 Préliminaires d'une analyse fonctionelle des pointes microlithiques de Bergumermeer /Pays-Bas/. Bolletin de la Société Préhistorique Française 75/2, 37-49.

PEÑALVER IRIBARREN, X., MUJIKA ALUSTIZA, J.A.

2003 Suelo de ocupación magdaleniense en la cueva de Praile Aitz I (Deba, Gipuzkoa): evidencias de arte mobiliar. Veleia 20, 157-181.

PIÉ BATLLE, J., VILA MITJÀ, A.

1991 Relaciones entre objetivos y métodos en el estudio de la industria lítica, en MORA, R.; TERRADAS, X.; PARPAL, A. \& PLANA, C. (eds.). Tecnología y cadenas operativas liticas. 271-278. (Treballs d'Arqueologia; 1). 
PLISSON, $\mathrm{H}$.

1985 Étude fonctionnelle des outillages lithiques préhistoriques par l'analyse des micro-usures; recherché méthodologique et archéologique. Thèse de Doctorat. Université de Paris I.

PLISSON, H., GENESTE, J.M.

1989 Analyse technologique des pointes à cran solutréennes du Placard (Charente), du Forneau du Diable, du Pech de la Boissière et de Combe-Saunière (Dordogne). Paléo 1, 65-105.

PRASLOV, N.D.

1972 Nekotorie spetzificheskie formi kamennij orudii Muralovskoi paleoliticheskoi stoianki. KSIA 131. Moskva.
ROGACHEV, A.N

1955 Kostionki IV -poslednie drevnekamennogo veka na Donu. MIA 45. Moskva.

SEMENOV, S.A.

1957 Pervobitnaya Tejnika. Materiali y Isledovania po Arjeologii SSSR 54. Moskva.

1964 Prehistoric Technology. Cory, Adams and Mackñay. London.

1981 Tecnología Prehistórica (Estudio de las herramientas y objetos antiguos a través de las huellas de uso). Akal Editor. Madrid.

VERHEYLEWEGHEN, J.,

1951 La lamelle magdalénienne à dos rabattu et son utilisation. Bolletin de la Société Préhistorique Française 48, 354-364. 
